\title{
O sistema carótico do encéfalo do Cebus apella sp., Linnaeus, 1766
}

\author{
Jussara Rocha Ferreira ${ }^{1,3}$, Irvênia Luiza de Santis Prada ${ }^{2}$ \\ ${ }^{1}$ Campus Universitário Darcy Ribeiro, Faculdade de Medicina Área de Morfologia, \\ Universidade de Brasília - UnB, \\ BC-321, Asa Norte, CEP 70910-900, Brasília, DF, Brasil \\ ${ }^{2}$ Faculdade de Medicina Veterinária e Zootecnia - FMVZ, \\ Universidade de São Paulo - USP, \\ Cidade Universitária, São Paulo, SP, Brasil \\ ${ }^{3}$ Autor para correspondência: Jussara Rocha Ferreira, jussararocha@unb.br
}

FERREIRA, J.R. \& PRADA, I.L.S. 2009. The carotid encephalic system of the Cebus apella sp., Linnaeus, 1766. Biota Neotrop. 9(1): http://www.biotaneotropica.org.br/v9n1/en/abstract?shortcommunication+bn03209012009.

\begin{abstract}
The carotid system of the encephalon of thirty species of the primate Cebus apella sp. (Linnaeus, 1766) was studied in this research. The material was collected according to international bioethics parameters and animals' welfare. The arterial system was perfused (water at $40^{\circ} \mathrm{C}$ ), injected with colored latex (Neoprene 450 , Dupont ${ }^{\circledR}$ do Brasil and Sulvinil ${ }^{\circledR}$ coloring, Glassurit), fixed in formaldehyde aqueous solution (10\%) and dissected under magnifying glass (RASOR, II-20) for the systematization of vessels. The carotid system is composed by the intracranial and internal portions of both right and left carotid arteries, the carotid arteries of the encephalon and their lateromedial and anteromedial branch divisions: the medial and rostral cerebral arteries, communicating caudal and interconnected arteries. It is interconnected through the caudal communicating arteries to the vertebral-basilar system $(100 \%)$. The internal carotid artery ends up being divided into three: caudal communicating arteries, medial cerebral and rostral cerebral $(50 \%)$ or into two: medial cerebral and rostral cerebral arteries (50\%). The medial cerebral artery is the most stable branch of this circuit. and after ventricularizing the olfactory bulbs (93.3\%), the rostral cerebral artery becomes anastomosis with the contralateral artery, forming the interhemispheric artery that connects the blood layers of the arterial circuit of the encephalon base. It can be considered that Cebus apella sp. depends on two supply sources to the encephalon: the basilar and carotid systems, what indicates an important degree of evolutionary maturity. The absence of anomalies in this primate demonstrates evolutionary stability. Keywords: encephalon, carotid system, cerebral arteries, neotropical primate Cebus apella sp.
\end{abstract}

FERREIRA, J.R. \& PRADA, I.L.S. 2009. O sistema carótico do encéfalo do Cebus apella sp., Linnaeus, 1766. Biota Neotrop. 9(1): http://www.biotaneotropica.org.br/v9n1/pt/abstract?shortcommunication+bn03209012009.

Resumo: Estudamos em trinta espécimes do primata Cebus apella sp. (Linnaeus, 1766) o sistema carótico do encéfalo em animais coletados dentro dos parâmetros internacionais de bioética e bem estar animal. O material teve o sistema arterial perfundido (água a $40^{\circ} \mathrm{C}$ ), injetado com látex corado (Neoprene 450, Dupont ${ }^{\circledR}$ do Brasil e Sulvinil ${ }^{\circledR}$ Corante, Glassurit), fixado em solução aquosa de Formaldeído (10\%) dissecado sob lupa (RASOR - II - 20) para sistematização dos vasos. O sistema carotico é composto pela porção intracraniana e intradural dos seguimentos dependentes das artérias carótidas internas esquerda e direita, as artérias carótidas do encéfalo (ace) e seus ramos de divisões látero-mediais e ântero-mediais: as artérias cerebrais médias acm e rostrais (acr), comunicantes caudais e artérias corioídeas Está interligado, através das artérias comunicantes caudais, ao sistema vértebro-basilar (100\%). A ace termina trifurcando-se (50\%), ou bifurcando-se (50\%) em arranjos vasculares que envolvem as acm e acr, acc e artéria corioídea. A acm é o ramo mais estável deste circuito e a acr após vascularizar os bulbos olfatórios $(93,3 \%)$ anastomosa-se com a artéria contralateral formando a artéria inter-hemisférica que conecta rostralmente os leitos sanguíneos do circuito arterial da base do encéfalo. Pode-se considerar que o Cebus apella sp. depende de duas fontes de suprimento para o encéfalo: os sistemas basilar e carotídico, isto indica um grau elevado de maturidade evolutiva. A ausência de anomalias demonstra neste primata estabilidade evolutiva.

Palavras-chave: cérebro, primata neotropical Cebus apella sp., artérias cerebrais, sistema carotídico. 


\section{Introdução}

Desde tempos imemoriais os homens buscam conhecer as formas vivas na tentativa de desvendar mistérios. Os primatas, em razão de sua similaridade física e relativamente comportamental com os humanos, tem sido objeto de vários estudos morfológicos enfocando o sistema vascular (De Vriese 1905, Elze 1910, Shellshear 1927, Watts 1934a, b, Miraglia \& Teixeira 1960, Souza et al. 1962, Weinstein \& Hedges 1962, Kassel \& Langfitt 1965, Ferreira \& Prada 2001, Silva \& Ferreira 2002a,b, Siqueira Neto \& Ferreira 2002, Silva \& Ferreira 2003, Ferreira \& Prada 2003, Silva et al. 2003, Pires \& Ferreira 2004a, b, Silva \& Ferreira 2005, Ferreira et al. 2005).

O circuito arterial da base do encéfalo interpretado por alguns autores (Kramer 1912, Shellshear 1927, Rogers 1947) como uma anastomose de irrigação foi analisado em primatas humanos, não humanos e em outros vertebrados, tendo seus componentes descritos com detalhes: nos ungulados, em cavalo (Santos 1987); em fetos de bovinos azebuados (Melo 1996); em suínos (Ferreira 2002); em búfalos (Ding et al. 2007); nos carnívoros, em cães (Alcântara 1992) e raposas (Depedrini \& Campos 2003); nos roedores, em ratos (Andrade 1983) e em capivaras (Reckziegel et al. 2004), além dos marsupiais (Lindermam et al. 2000). A respeito de variações de características morfológicas de vasos que compõem o circuito arterial da base do encéfalo, foram estudados temas como a frequência da complexão ou não deste circuito (Fawcett \& Blanchford 1905-1906); a direção da circulação em áreas funcionais e a pressão do fluxo sanguíneo em relação ao diâmetro dos vasos (Shellshear 1927, Himwich \& Clark 1968) além de outros aspectos considerados, como a subdivisão do circuito em dois segmentos referidos por Krishnamurti (1968), quais sejam: o circuito vértebro basilar e o carótico.

$\mathrm{Na}$ tentativa de esclarecer aspectos da vascularização cerebral em primatas neotropicais, neste caso o macaco prego (Cebus apella sp., Linnaeus, 1766), este estudo ateve-se à descrição do sistema carótico do encéfalo, enfocando seus vasos formadores, as subdivisões que compõem a porção rostral do circuito arterial além de fazer referência a interligação deste sistema com o sistema vértebro - basilar.

Entende-se que conhecer o binômio forma - função de animais da biodiversidade (savanas e florestas) tropical é uma maneira de contribuir para a preservação das espécies que nelas vivem. Embora o Brasil possua o maior estoque de primatas do planeta vivendo em condições naturais, muito ainda há que se estudar para garantir a potencial capacidade de existir desta fauna, visto que nunca se destruiu tanto a vida animal como neste último século.

\section{Material e Método}

Esta pesquisa é quantitativa, e descreveu a formação do sistema carótido do encéfalo do macaco prego (Cebus apella sp., Linnaeus, 1766) em trinta espécimes, tendo sido estes reaproveitados de pesquisas autores, conforme prevê o principio do $3 \mathrm{R}$, coletados dentro das regras internacionais de bioética, com o suporte da Faculdade de Medicina Veterinária e Zootécnica da Universidade de São Paulo (FMVZ / USP). O material foi recebido a fresco (animais que vieram a óbito na Fundação Zoológico de São Paulo e que mediante convênio foram remetidos à FMVZ para fins de ensino e pesquisa), tendo sido o sistema arterial perfundido (água a $40^{\circ} \mathrm{C}$ ), injetado com látex corado (Neoprene ${ }^{\circledR} 450$, Dupont ${ }^{\circledR}$ do Brasil e Sulvinil ${ }^{\circledR}$ 2350-0003, Glassurit), fixado com solução de formaldeído (10\%) e dissecado sob lupa (RANSOR, II-20). Na seqüência, foi feita a sistematização, descrição e documentação dos vasos.

Para fins de quantificação considerou-se 30 indivíduos, sendo que em cada um foram analisados os antímeros direitos e esquerdo (60 casos) como dados isolados. Tomou-se como parâmetro estudos (Bugge 1974, Guerin et al. 1976) nos quais as variantes anatômicas observadas entre os antímeros foram atribuídas ao brotamento das artérias de somitos contra-laterais como dados independentes durante o período embrionário.

$\mathrm{Na}$ organização e análise dos resultados, a denominação dos vasos observou a normatização da Nomenclatura Anatômica Veterinária (1994).

\section{Resultados}

Descrição do sistema carotídico em Cebus apella sp. (C. apella.): compõem o sistema carótico do encéfalo (segmento rostral do polígono de Willis), parte da porção intracraniana das artérias carótidas internas (aci) direita e esquerda. Esta parte do circuito arterial da base do encéfalo nesta espécie é formada pela sequiência dos segmentos e dos ramos de divisão dependentes de porção intadural das aci de ambos os lados: as artérias carótidas do encéfalo (ace), cerebrais médias (acm) e cerebrais rostrais (acr). Os segmentos rostais dependentes destes vasos, em cada antímero, circundam, caudo-rostralmente, a região anterior à base dos pedúnculos cerebrais, bem como as regiões infundibular e quiasmática. No ponto de formação do tracto óptico, a acr se destaca como o vaso de continuação da ace e se inclina em direção a linha mediana, indo se anastomosar com a artéria do antímero oposto, originando a artéria interhemisférica. O sistema carótico do encéfalo em $100 \%$ das observações é interligado ao sistema vértebro - basilar através de uma anastomose de irrigação entre os dois leitos arteriais: as artérias comunicantes caudais (acc), nos dois antímeros (Figura 1).

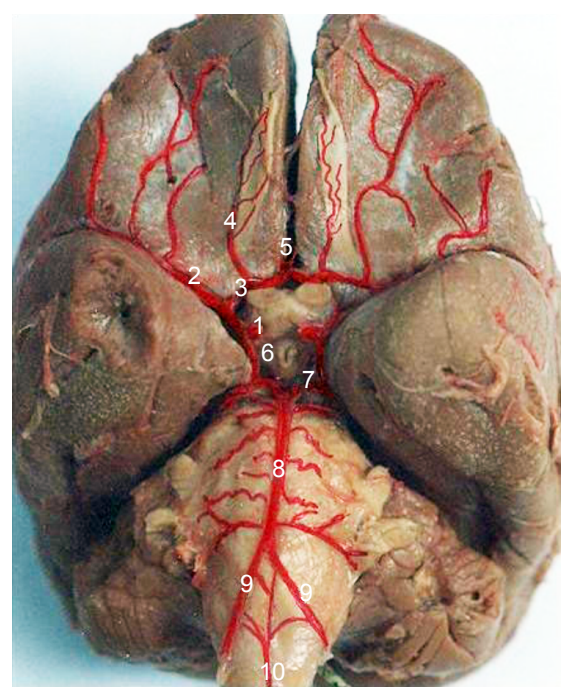

Figura 1. Fotografia da vista ventral do encéfalo do primata Cebus apella sp. (Linnaeus, 1766) destacando-se os vasos componentes do sistema carótico do encéfalo nos dois antímeros, as artérias: carótidas do encéfalo (1); cerebrais médias (2); cerebrais rostrais (3); e olfativas (4). A finalização da porção rostral do circuito carotídico: artéria interhemisférica (5). A interligação entre os sistemas carotídico e vértebro-basilar: artérias comunicantes caudais (6). Os vasos componentes do sistema vertebro-basilar, as artérias: cerebrais caudais (7); basilar (8); vertebrais direita e esquerda (9); e espinhal ventral (10).

Figure 1. Photograph of the ventral view of the Cebus apella primate encephalon (Linnaeus, 1766) emphasizing the vessels that compose the carotid system of the encephalon in both antimers, the arteries: the encephalon carotids (1), intermediate cerebral (2), rostral cerebral (3) and olfactory (4). Completion of the rostral portion of the carotid circuit: inter-hemispheric artery (5). The interconnection between the carotid and vertebral-basilar systems: caudal communicating arteries (6). The vessels that compose the vertebral-basilar system, the arteries: caudal cerebral (7), basilar (8), right and left vertebral (9) and ventral spinal (10). 
A ace e seus ramos nos seguimento intradural, na base do encéfalo, e sustentada pela meninge piamater, quer esteja situada sobre as circunvoluções cerebrais, ou na profundidade dos sulcos do cérebro sobre e sob os quais se distribuem.

Artéria carótida interna: as ace direita e esquerda penetram no crânio atravessando o canal carótico no osso temporal. Neste estudo nos ativemos em observar os seguimentos derivados desta a partir do ponto em que estes perfuraram a duramáter e a aracnóide, abordando a base do encéfalo lateralmente ao quiasma óptico nos dois antímeros.

Observou-se que existe um pequeno segmento intradural do vaso, entre 2 e $4 \mathrm{~mm}$ que ao se aproximar dos hemisférios cerebrais, de ambos os lados, aborda o circuito arterial da base do encéfalo em sentido caudo-rostral. Neste trajeto a artéria foi denominada de ace e, antes de sua terminação, aconteceram particularidades relativas à: emissão de ramos colaterais; presença de anastomoses e no modo de terminação desta, assim verificados: as ace emitem como ramos colaterais as artérias cerebrais caudais (acc), fato este que ocorreu em 46,6\% dos espécimes, 12 vezes nos dois antímeros e 4 vezes à esquerda (Figura 2a e d); as ace, na base do encéfalo, fornecem ramos terminais, sendo que esta terminação pode exibir a característica de uma trifurcação (Figura 2b), da qual participaram os vasos: acm, acr e acc. A ace após originar a acc no antímero correspondente ainda forneceu, antes de sua terminação, um ramo colateral dirigido dorsocaudalmente em relação ao soalho dos ventrículos laterais, a artéria corioídea (aco), fato este observado em 11,6\% dos casos (Figura 2d), 2 vezes à direita, 2 vezes nos dois antímeros e uma vez no antímero direito. A ace, após a origem da acc, terminou trifurcando-se em outro arranjo constituído pelos seguintes vasos: acm e acr e aco (Figura 2d).

Pôde-se notar que no trajeto das ace, e dos primeiros segmentos das artérias cerebrais médias e rostrais, curtos e sinuosos que se dirigem à base do encéfalo e à superfície ventral dos hemisférios cerebrais correspondentes: os capilares quiasmaticos. Estes capilares formam uma extensa rede vacular ao redor da região da hipófíse e da base do quiasma óptico.

A ace apresentou-se com calibre ligeiramente inferior ao da artéria basilar, exibindo simetria entre os antímeros direito e esquerdo em todas as observações (Figura 1).
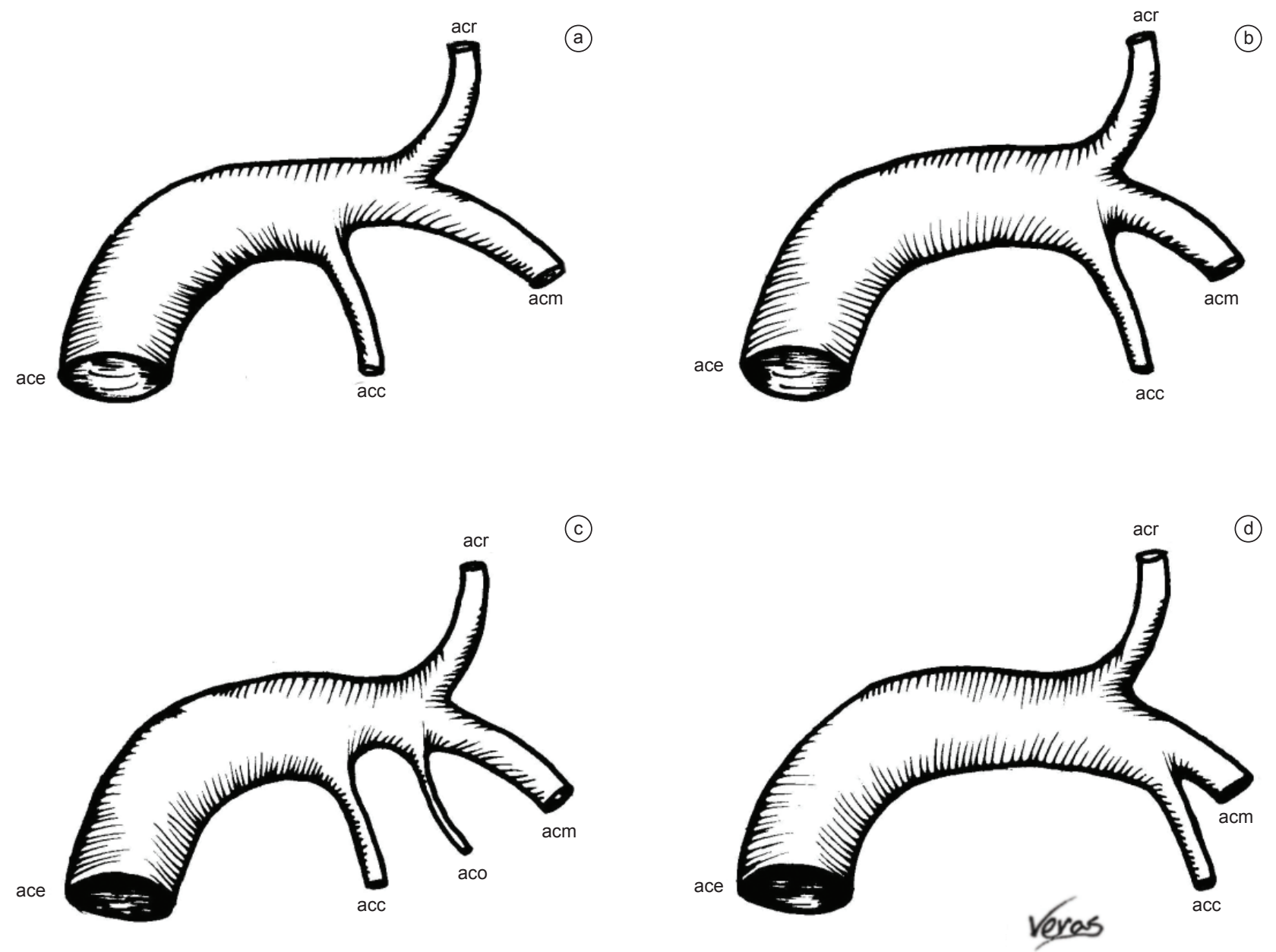

Figura 2. Em a, b, c e d estão representadas esquematicamente as variantes anatômicas da subdivisão terminal dos ramos intracranianos e intradurais da artéria carótida no encéfalo de Cebus apella sp. (Linnaeus, 1766) : artéria carótida do encéfalo (ace), artéria cerebral média (acm), artéria cerebral rostral (acr), artéria comunicante caudal (acc) e artéria coroídea (aco). a e c) terminação da ace por bifurcação; b e d) terminação da ace por trifurcação.

Figure 2. a, b, c and d show the schematically representation of the anatomical variations of the terminal division of intradural and intracranial branches of the carotid artery in the encephalon: carotid artery of the encephalon (cae), intermediate cerebral artery (ica), rostral cerebral artery (rca), caudal communicating artery (cca) and choroidal artery (cha). a and c) termination of cae by bifurcation b and d, termination of cae by trifurcation. 
A acm foi identificada nos 30 espécimes, de disposição par, calibre constante e relativamente simétrico, representando o ramo terminal mais calibroso de continuação da ace. Percorre o sulco lateral do cérebro em toda a sua extensão, distribuindo ramos que vascularizam a maior parte da face infero-lateral de cada hemisfério cerebral. Sua origem ocorreu por bifurcação (50\%; Figura 2a e c) ou trifurcação (50\%; Figura $2 \mathrm{~b}$ e d) terminal da ace. Quando originada por bifurcação, está se deu através de tronco arterial formado pelas artérias referidas e pelas acr ou acc, ou seja, três vasos em arranjos da dois. Teve-se a oportunidade de detectar as seguintes variáveis entre os antímeros: artérias bifurcando-se bilateralmente (43,3\%); somente no antímero esquerdo (6,6\%). Quando a origem aconteceu por trifurcação $(50 \%)$ as artérias envolvidas foram as próprias acm, juntamente com as acr e acc, além das aco, ou seja, quatro vasos combinados em arranjos de três.

$\mathrm{Na}$ trifurcação em que participaram as acm e acr e acc (40\%), pôde-se constatar que estas variantes obedeceram a seguinte disposição: origem em tronco triplo bilateral, identificada em 36,7\% dos casos; origem em tronco triplo à direita; em 3,3\%. Na trifurcação em que participaram as acm, acr e aco, $(10 \%)$, as variações entre os antímeros estiveram dispostas como segue: trifurcação bilateral $(3,3 \%)$; trifurcação à direita $(5 \%)$ e trifurcação à esquerda $(1,6 \%)$.

Vale registrar que a aco se destacou como o ramo colateral principal da acm em seu trajeto inicial exibindo os seguintes arranjos: esteve presente bilateralmente $(56,6 \%)$, unilateralmente à esquerda $(23,3 \%)$ e unilateralmente à direita $(6,6 \%)$.

Destaca-se também que, de ambos os lados, em um espécime as acm tiveram origem comum com as acc e em outro caso o mesmo fato ocorreu apenas no antímero esquerdo.

A artéria cerebral rostral (acr): é o ramo terminal rostral de subdivisão das artérias carótidas do encéfalo direita e esquerda (Figura 1). No que se refere à sua origem esta aconteceu por bifurcação $(50 \%)$ ou por trifurcação (50\%) da ace. Em sua origem relaciona-se medialmente com o quiasma óptico na linha da inserção do infundíbulo da hipófise, dirige-se e encurva-se rostralmente em relação à linha mediana, passando dorsalmente aos tractos ópticos e caudalmente aos bulbos olfatórios. Neste trajeto descreveu uma curvatura, suja concavidade rodeia e delimita a porção média da base do encéfalo. Após emitir as artérias olfativas, a artéria cerebral rostral encaminhou-se em direção à artéria homônima, no antímero oposto, com a qual anastomosou-se por inosculação dando origem à artéria inter-hemisférica.

Com relação ao trajeto, pode-se inferir que as artérias cerebrais rostrais e seus ramos compuseram a maior parte dos vasos que formam o segmento rostral do circuito arterial da base do encéfalo, vasos, em muitos casos, sinuosos e ligeiramente irregulares entre os antímeros. Da face cranial das acr, de ambos os lados, partem ramos finos distribuídos sob a base do encéfalo, a região quiasmática e aos nervos ópticos. Na continuidade do trajeto da acr, de cada lado do circuito arterial, percebe-se a presença de um a três ramos colaterais delgados distribuídos na face ventral da porção rostral dos hemisférios cerebrais e da região olfatória (Figura 3). Pouco antes de sua finalização, a acr emitiu a artéria olfativa $(93,3 \%)$, de fino calibre, dirigida longitudinalmente à face inferior do lobo olfatório correspondente (Figura 3).

A anastomose por convergência do seguimento rostral das acr entre os antímeros deu origem ao tronco interhemisférico com as seguintes variações, nos últimos $3 \mathrm{~mm}$ de trajeto: em 4 antímeros as acr convergiram para a linha mediana anastomosando-se por inosculação, sem emitir colaterais nos planos lateral e ventral (6,6\%); em $46,6 \%$ das observações, bilateralmente no trajeto em direção à linha mediana (Figura 3), as acr emitiram um ramo colateral dirigido à região olfatória, a artéria olfativa. No plano mediano, as acr anastomosaram-se por convergência, formando a artéria interhemisférica

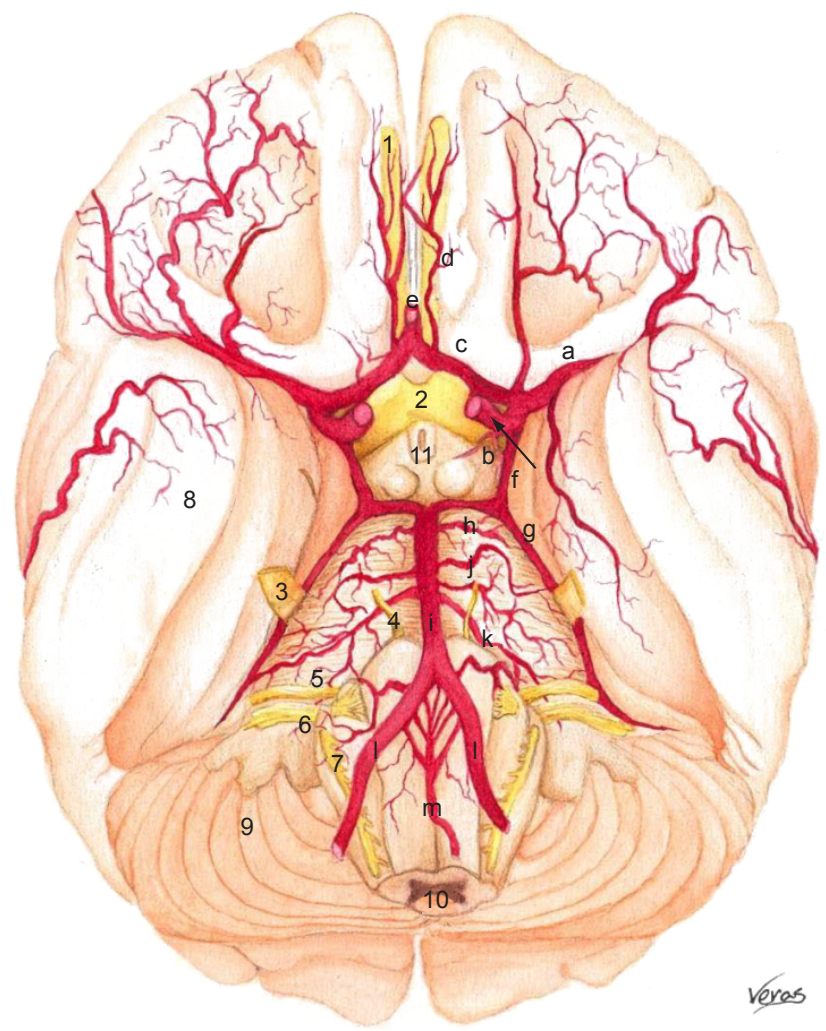

Figura 3. Representação esquemática das artérias e nevos da base do encéfalo do Cebus apella sp. (Linnaeus, 1766), destacando-se no sistema carotídico (antímero esquerdo) as artérias: carótida do encéfalo $(\rightarrow)$, cerebral média (a); corioídea (b); cerebral rostral (c); olfativa (d); inter-hemisférica (e); comunicante caudal (f). Estão representadas algumas artérias, do sistema vértebro basilar: cerebral caudal $(\mathrm{g})$; cerebelar rostral (h); basilar (i); pontinas (j); cerebelar caudal (k); vertebral esquerda e direita (l); espinhal ventral (m). No antímero direito estão representadas algumas estruturas e as relações das artérias com a origem aparente de alguns (n) cranianos: bulbo olfatótio (1); quiasma óptico (2); n. trigêmio (3); n. abducente (4); n. facial e vestíbulo coclear (5); n. glossofaríngeo e vago (6); n. acessório (7); hemisfério cerebral direito, lobo temporal (8); hemisfério cerebelar direito (9); medula espinhal (10); infundíbulo da hipófise (11).

Figure 3. Schematic representation of the arteries and nerves of the base of the Cebus apella encephalon (Linnaeus, 1766), emphasizing the carotid system (left antimer) the arteries: carotid artery of the encephalon $(\rightarrow$ ), intermediate cerebral (a), carotid (b), rostral cerebral (c), olfactory (d), inter-hemispheric (e) caudal communicating (f). Some arteries of the vertebral basilar system are represented: caudal cerebral $(\mathrm{g})$, rostral cerebellar (h), basilar (i), pontine (j), caudal cerebellar (k), left and right vertebral (l), ventral spinal (m). The right antimer show some structures and the relationships of the arteries with the apparent origin of some (n) cranial: olfactory bulb (1), optic chiasm (2) n. trigeminal (3) n. abducent (4) n. facial and cochlear vestibule (5) n. glossopharyngeal and vagus (6), n. accessory (7) right cerebral hemisphere, temporal lobe (8), right cerebellar hemisphere (9), spinal cord (10), the pituitary infundibulum (11).

(100\%), cujo calibre equivaleu relativamente à somatória dos vasos formadores (acr direita e esquerda). Foi identificada em 31,6\% da amostra a presença de outro ramo colateral inominado da acr, além da artéria olfativa. Este vaso dirigiu-se à superfície inferior da região frontal dos hemisférios cerebrais correspondentes e piamater adjacente estando presente bilateralmente: (13,3\%); à direita (11,6\%); bilateralmente e com calibre superior ao da artéria olfativa $(6,6 \%)$. Foram também registrados em $10,0 \%$ dos casos a presença de duas 
artérias colaterais para a região inferolateral e meníngea dos hemisférios cerebrais adjacentes antes da emergência das artérias olfativas, como segue: à direita $(5,0 \%)$ e à esquerda $(5,0 \%)$. Pode-se ressaltar que em dois casos um vaso se destacou lateralmente e paralelamente, em relação à acm, dirigindo-se ao território de vascularização desta, comportando-se como uma artéria colateral de calibre considerável. Em uma observação $(1,6 \%)$ foram vistas três artérias colaterais no antímero esquerdo, além da constante artéria olfativa que, neste caso, nos dois antímeros apresentou-se bastante sinuosa e de calibre mais significativo que o de costume.

No espécime 25, no antímero direito, a acr anastomosou-se com a acm através de um ramo colateral arqueado. Pode-se registrar, na observação 20, hipoplasia da acr, no terço inicial de sua origem no antímero esquerdo. Após a emergência de uma colateral paralela à acm, o tronco principal da acr aumentou de volume e continuou seu trajeto com calibre equivalente ao da artéria do antímero oposto até anastomosar-se com esta.

\section{Discussão}

O sistema carótico é representado pela continuação das duas artérias carótidas internas, uma direita e uma esquerda que se encarregam de irrigar grande parte do encéfalo do C. apella, no qual as artérias nominadas como artérias carótidas do encéfalo (ace), no trajeto intradural, antes de sua terminação, tem algumas particularidades que comentaremos.

A maneira mais usual (50\%) deste segmento da ace se comportar foi após perfurar a duramáter, na extremidade do sulco lateral, se dividir em artérias cerebral média, cerebral rostral e comunicante caudal. Além deste, foram ainda observados outros três arranjos, sendo que em um deles, a ace, após perfurar a duramáter, forneceu seu primeiro ramo: a artéria comunicante caudal $(46,6 \%)$; ou dois ramos: a artéria comunicante caudal e a artéria coroídea para, a seguir, terminar bifurcando-se em artérias cerebral média e rostral (10\%); no terceiro arranjo a ace após perfurar a duramáter forneceu seu primeiro ramo: a artéria comunicante caudal e a seguir terminou se trifurcando em artérias cerebral média, cerebral rostral e coroídea (1,6\%). Alguns autores destacam artérias carótidas de pequeno tamanho no seu trajeto intracraniano, ou seja: no orangotango com 4 ou $5 \mathrm{~mm}$; nos Cercopithecidae, 3 ou 4 mm (Watts 1934b); no macaco rhesus, após penetrar na duramáter e fornecer a artéria oftálmica, $5 \mathrm{~mm}$ (Weinstein \& Hedges 1962). São iguais no homem e no chimpanzé (Sperino 1897); são iguais no homem e no gibão (De Vriese 1905); e correm no seio cavernoso por $4 \mathrm{~mm}$ no macaco rhesus (Weinstein \& Hedges 1962, Castelli \& Huelke 1964), nesta amostra a ace corresponde à artéria apontada pelos autores e mediu entre 2 e $4 \mathrm{~mm}$.

Castelli \& Huelke (1964) chamam a atenção para a análise deste segmento da artéria carótida interna em seu trajeto intracraniano que vai desde o momento em que o vaso, após alcançar a entrada do canal carotídico, corre dentro do osso temporal, para a seguir emergir na base do crânio, atravessando o seio sigmóide e dirigindo-se ânteromedialmente à região quiasmática no macaco rhesus Este autores sustentam a hipótese de que mudanças ocorridas no vaso durante a filogenia representam um fator importante na classificação dos primatas. A morfologia destes vasos evidencia que: a passagem do sangue para o cérebro tem modelo distinto nos primatas vivos conforme Conroy \& Packer (1981) e Feagle (1988). O principal suprimento sanguíneo para a cabeça dos primatas, analisado sob o ponto de vista das relações filéticas de primatas vivos e fósseis, provém dos seguintes vasos: nos símios, as artérias carótidas internas e as vertebrais; no gibão, as artérias são iguais às do homem, isto é, as carótidas internas e vertebrais (De Vriese 1905); nos mamíferos, as artérias carótidas externas e internas e a artéria estapedial (Dyrud 1944); nos Leptictidis, as artérias promontorial e estapedial (Mckenna 1966). Em outros vertebrados: o sistema carótico externo-interno e o sistema estapedial (Bugge 1974); as artérias carótidas internas direita e esquerda e artérias vertebrais, sendo que as primeiras, em alguns modelos, penetram na cavidade craniana como duas artérias distintas, quais sejam, a artéria estapedial e a artéria promontorial (Feagle 1988). No esclarecimento das questões sobre as artérias carótidas em seu curso inicial alguns autores destacaram que: o entendimento das diferenças entre o sistema arterial cefálico de insetívoros, primatas, roedores e lagomorfos são esclarecidos com base em dados de embriogênese humana (De La Torre \& Netsky 1960 apud Bugge 1974); o sistema arterial carótico externo-interno desenvolve-se de um estágio ontogenético, remoto dos primitivos arcos aórticos (Dyrud 1944). Foi estudada a relação da artéria carótida com os nervos vago, hipoglosso, acessórios e glossofaríngeo em primatas (Stepsirhine) por Conroy \& Packer (1981), onde as autores estabeleceram relação de homologia entre este feixe, artéria- nervos, na região promontorial, como sendo diferente nos Lemuos e primatas indicando mudanças ocorridas na mofologia durante o período eoceno.

O trabalho de Bugge (1974), com especial referência às questões ontogenéticas filogenéticas, e taxomônicas, descreveu um modelo básico primitivo, abrangendo o sistema arterial carótico externo-interno e o sistema estapedial. Estudos morfológicos evidenciam que estes dois sistemas passaram por modificações durante a embriogênese, e que podem se apresentar, ou como uma variação do número de artérias ou pela presença de seis anastomoses. Ao analisar os aspectos dos vasos no período de anastomoses, ou segundo período morfogenético, no qual o padrão vascular do cérebro foi definitivamente estabelecido, estas modificações foram confirmadas por Vieira (1981) ao estudar o modelo humano. Outros autores destacaram que o modelo do suprimento sanguíneo cefálico arterial é primitivo ou avançado na medida em que ele se afasta ou se aproxima do modelo padrão, ao qual é atribuída a representação mais primitiva tanto na ontogenia quanto na filogenia. Os leptictids retiveram um modelo primitivo na região da orelha no período terciário (Mckenna 1966); nos carnívoros atuais há uma predominância da artéria carótida externa, sendo que os gatos apresentam obstrução proximal completa da artéria carótida interna compensada por três anastomoses (Dyrud 1944). Nos Cebídeos (Aotus trivirgatus) todo o tronco da artéria estapedial é obliterada, nos Marmosets (Saguinus midas) a parte proximal do tronco estapedial e a parte média do tronco supraorbital são obliterados (Bugge 1974). O suprimento feito pelos ramos promontorial e estapedial da artéria carótida interna têm os seguintes modelos de suprimento: nos lêmures, artéria estapedial; tarsus e macacos do novo mundo, ramo estapedial da artéria carótida interna; Show loris, ramo faringeal ascendente da artéria carótida interna macacos e grandes primatas, ramo promontorial da artéria carótida interna (Feagle 1988). A forma encontrada nos vasos da base do C. apella, considerada a partir do modelo padrão básico de Bugge (1974) permitiu a considerar que este animal se enquadra entre aqueles nos quais o sistema promontorial desapareceu, pois a artéria carótida externa não participa do suprimento do encéfalo e as artérias carótidas internas representam uma modificação das antigas artérias estapediais. Paralelamente a isto, as artérias vertebrais se desenvolveram, sendo que três anastomoses mantiveram-se, comparado o padrão básico com o atual. Isto foi confirmado em Cebideos (Aotus trivirgatus), por Bugge (1974).

Tratando da parte rostral do circuito arterial da base do encéfalo do C. apella está se propondo que entram nesta composição os ramos de divisão rostro-mediais derivados das artérias carótidas internas direita e esquerda, cujo seguimento intradural foi denominado neste estudo de artéria carótida do encéfalo e é deste segmento que depende o que se entende por sistema carotidico rostral. Difere desta proposta achados (Ferreira \& Prada 2001, Ferreira \& Prada 2003) que trataram 
das acm, acr e aco, como ramos de divisão da artéria carótida interna. Outros estudos (De Vriese 1905) argumentam que as aci do homem são iguais às do chimpanzé (Sperino 1897) ou iguais às do gibão. Artérias carótidas internas dividindo-se em dois ramos terminais são contempladas no chimpanzé (Shellshear 1930); no Cereophitecidae e Cebidae (Watts 1934b); no macaco rhesus (Dyrud 1944, Lineback 1961, Weinstein \& Redges 1962); no gorila (Raven \& Hill 1950); no homem (Testut \& Latarjet 1954) e nos Cebídeos Hill (1960) aposta morfologia semelnhante a este estudo no $C$. apella. A ace no curto trajeto intradural apresentou-se, nos espécimes, com calibre ligeiramente inferior ao da artéria basilar (isto se comparadas na mesma observação), dado este que pode ser confrontado com o apontado por Watts (1934b), segundo o qual a artéria é referida como pouco calibrosa no macaco rhesus e $8 \mathrm{~mm}$ menos calibrosa do que a artéria carótida externa nos Cebídeos. Estes dados confirmaram ramos terminais das ace representados por uma bifurcação (50\%) ou por uma trifurcação. Quando ocorreu bifurcação os vasos envolvidos foram: as acm e acr, bilateralmente (43,3\%) e somente no antímero esquerdo $(6,6 \%)$. Quando ocorreu trifurcação, os vasos envolvidos foram as artérias cerebrais médias e rostral, a comunicante caudal e a coroídea. $\mathrm{O}$ tronco triplo entre as três primeiras artérias anteriormente referidas ocorreu em $40 \%$ destes espécimes, nos dois antímeros ou apenas no antímero direito. Nos outros $10 \%$ os vasos envolvidos na trifurcação foram as artérias coroídeas, além das duas cerebrais rostral e média, bilateralmente, à direita e à esquerda.

A divisão da artéria carótida interna em dois ramos foi apontada nos Cebídeos por Hill (1960), mas, com relação à bifurcação final da artéria carótida interna fica a dúvida se a literatura está se referindo (quando faz comentários gerais) ao primeiro (extradural) ou ao segundo (intradural) segmento da artéria carótida interna após a sua penetração no interior da cavidade craniana. Para os espécimes objeto deste estudo ficou claro que a artéria carótida interna de ambos os lados, penetrando no crânio tem um trajeto extradural que não analisamos, antes da sua penetração para o interior da duramater e um trajeto intradural, sendo que este último, somente, esteve envolvido com os quatro vasos que descrevemos. O primeiro, a artéria coroídea, é simplesmente um ramo colateral (Ding et al. 2007) confirmam o mesmo no Bos grunniens e Reckzieget et al. e Hydrochoerus hydrochaeris; o segundo, a artéria comunicante caudal, pode ter duas interpretações, ou é uma colateral, ou é uma anastomose; os dois últimos, as artérias cerebrais média e rostrais são ramos terminais conforme estudos recentes confirmaram (Ferreira \& Pires, 2004). Com relação a estes achados não percebemos na revisão da literatura, resultados que pudessem confrontar com estes dados. A análise critica, deve considerar o que foi dito por De Vriese (1905): que muitas vezes as interpretações dos modelos ou padrões atuais de vascularização cerebral são considerados sem base ontogenética ou filogenética.

Se olharmos para o modelo atual do C. apella, a artéria comunicante caudal, do ponto de vista morfológico, em 50\% dos achados aparece como um dos ramos da divisão final (em trifurcação) da artéria carótida do encéfalo; no entanto, na outra metade dos achados pode-se interpretá-la como sendo uma colateral. De posse dos modelos morfológicos das 60 artérias do $C$. apella analisadas, em função das características baseadas no binômio forma-função, deve-se levar em conta a interpretação que privilegie aspectos mais amplos além dos morfológicos, quais sejam, os ontogenéticos e os filogenéticos, considerados por De Vriese (1905) seguida de outros autores, como Testut \& Latarjet (1954), Dyrud (1944), McKenna (1966), Lazortes apud Barbosa et al. (1969), Bugge (1974), Fleage (1988) e Ding et al. 2007. Estes estudos reforçam a hipótese aqui levantada: a acc pode ser considerada uma anastomose de irrigação entre os sistemas vertebral e carotídico.
A acm dentre os ramos de ace é o vaso mais estável filogeneticamente (Hill 1960). As considerações sobre esta artéria neste Cebídeo devem-se ao fato dela ter origem em tronco comum, seja com a acr, ou seja, com as acc e acr. Todavia, no C. apella a acm não participou da formação do circuito arterial da base do encéfalo, podendo ser interpretada como um ramo terminal de disposição constante e simétrica (100\%), e que após a sua emergência insinua-se no sulco lateral, percorrendo-o, para se subdividir nos hemisférios correspondentes.

Um dos ramos colaterais da acm, a aco, teve sua origem também em outros vasos envolvidos na composição dos segmentos do circuito arterial da base do encéfalo constatada como trifurcação: terminal da ace (10\%); como ramo colateral da acm bilateralmente $(56,6 \%)$, unilateralmente à esquerda $(23,3 \%)$, e unilateralmente à direita $(6,6 \%)$. Artérias coroídeas delgadas com trajeto caudal atingindo o prolongamento temporal dos ventrículos laterais foram vistas no sagüi (Souza et al. 1962), nos primatas humanos (Warwick \& Williams 1979) e nas capivaras (Reckziegel et al. 2004).

O ramo terminal rostral da ace é representado pela acr nos dois antímeros, exibindo percurso ântero medial em torno da porção média da base do encéfalo. Fato semelhante foi referido no orangotango (Watts 1934b). Nestes espécimes as artérias, se anastomosaram originando o tronco interhemisférico na linha mediana. Não emitiram colaterais nos planos laterais e ventrais $(6,6 \%)$; emitiram um ramo colateral para a região olfatória $(46,6 \%)$; destacou-se delas um vaso inominado dirigido para a porção inferior dos hemisférios correspondentes e pia-máter adjacente $(31,6 \%)$; emitiram duas artérias colaterais dirigidas à região ínfero lateral do lobo frontal e pia-máter adjacente (10\%). Não foi encontrado na literatura resultados que pudessem confrontar com estes achados.

Da porção terminal da acr derivaram-se ramos colaterais rostrais, a artéria olfatória, de calibre simétrico, fina e destinada à região inferior dos lobos olfatórios, podendo este vaso ser igualmente dependente da artéria interhemisférica conforme foi relatado em C. apella (Ferreira \& Pires 2004, Ferreira et al. 2005); artérias olfativas dependentes de ramos do circuito carotidico rostral foram também referidas por Allcântara (1996a, b) e Depedrini \& Campus (2003), em carnívoros e por Lindermam et al. (2000) em marsupiais. Shellshear (1927) tratando deste assunto no orangotango, admitiu que o segmento inicial da acr, até a região de emergência da artéria olfativa está relacionado com o sentido do olfato. De Vriese (1905), Gillilan (1969) e Testut \& Latarjet (1954) consideram que neste segmento as variáveis estão na dependência do estágio evolucionário do sentido do olfato, enquanto Puchardes-Orts et al. (1975) aventam a hipótese de que as variações no segmento pré-comunicante da artéria cerebral anterior no homem começam a surgir quando o cérebro olfativo humano começou a regredir.

O ramo terminal das acr resultou da união das artérias de ambos os lados em um vaso único no $C$. apella denominado de artéria interhemisférica descrita em detalhes por Ferreira et al. (2005). Esta anastomose fecha o circuito arterial da base do encéfalo no seu segmento rostral. Reportamos-nos, assim, ao considerado por De Vriese (1905) de que as acr, a partir dos répteis, se anastomosam entre os lados, formando uma artéria mediana ímpar que contorna o joelho do corpo caloso, ou estão unidas por uma série de anastomoses transversais. Artéria mediana ímpar é característica dos monotremos, marsupiais, edentatas, perissodáctilos, artiodáctilos, alguns roedores, insetívoros, quirópteros, pinípedes, lêmures e símios (De Vriese 1905), e aqui nós acrescentaríamos este Cebídeo. Artérias cerebrais anteriores unidas por comunicantes foram vistas no orangotango (Shellshear 1927, Watts 1934b). Artérias cerebrais anteriores anastomosando-se com a contra lateral foram apontadas no chimpanzé (Shellshear 1930) e em macacos do velho mundo, Macaca mulatta e Papio papio (Watts 1934a). 
Este estudo permitiu concluir que:

a) O sistema carótico do encéfalo do C. apella é dependente de duas fontes antiméricas de suprimento sanguíneo: as artérias carótidas internas direita e esquerda. A artéria carótida interna, de cada antímero, exibiu uma porção intradural, a artéria carótida do encéfalo, que antes de sua terminação deu origem às artérias comunicantes caudais e a seguir emitiu uma colateral, a artéria coroídea;

b) A ace terminou bifurcando-se (50\%) em acm e acr, ou trifurcando-se $(50 \%)$ em acm, acr e acc. A acm é o ramo mais estável $(100 \%)$ de bifurcação $(50 \%)$ ou trifurcação $(50 \%)$ terminal da ace, de calibre constante e simétrico entre os antímeros, sendo também a mais calibrosa no ponto de origem;

c) A acm, após destacar-se do tronco carótico emitiu um ramo colateral freqüente a artéria corioídea, bilateralmente $(56,6 \%)$, unilateralmente à esquerda $(23,3 \%)$ ou à direita $(6,6 \%) ; \mathrm{e}$

d) A acr é o ramo terminal (100\%) de divisão da ace, bifurcandose $(50 \%)$, concomitante com a acm, ou trifurcando-se (50\%), concomitante com as acm e acc. A acr, em seu trajeto, frequentemente $(93,3 \%)$ deu origem à artéria olfativa, em correspondência à face inferior do lobo olfatório correspondente. A acr termina anastomosando-se com o vaso contralateral, constituindo um tronco ímpar (100\%), a artéria interhemisférica, que se insinua na fenda sobre o corpo caloso além de conectar rostralmente os leitos sanguíneos que suprem as porções ântero laterais dos hemisférios cerebrais no C. apella.

\section{Agradecimentos}

Ao CNPq, Conselho Nacional de Desenvolvimento Cientifíco e Tecnológico, pelo apoio recebido e ao ilustrador científico Luiz Henrique Veras Neves, pela confecção dos esquemas.

\section{Referências Bibliográficas}

ALCÂNTARA, M.A. \& PRADA, I.L.S. 1996a. Artérias da base do encéfalo de cães (Canis familiaris, L., 1758). I. Estudo anatômico de suas origens e comportamento. Braz. j. vet. res. anim. sci. 33(2):67-71.

ALCÂNTARA, M.A. \& PRADA, I.L.S. 1996b. Artérias da base do encéfalo de cães (Canis familiaris, L., 1758). II. Formação e comportamento do circuito arterial da base do encéfalo. Braz. j. vet. res. anim. sci. 33(2):72-76.

ANDRADE, F.F. 1983. O circulo arterial da base do cérebro do rato de laboratório: contribuição para o seu estudo analítico e comparativo. Trabalho de síntese, no âmbito das provas de aptidão pedagógica e capacidade científica do Estatuto da Carreira Docente Universitária. Faculdade de Ciências Médicas da Universidade Nova de Lisboa, Lisboa.

BARBOSA, L.L., PURRIEL, J.A., MEERHOFF, W. \& MEDOC, I. 1969. El polligonno de Willis y sus variaciones. Acta neurol. latinoam. 15(4):224-236.

BUGGE, J. 1974. The cephalic arterial system in insetivores, primates, rodents and lagomorphs, with special reference to the sistematic classification. Acta anat. 87(suppl):1-160.

CASTELLI, W.A. \& HUELKE, D.F. 1964. The arterial system of the head and neck of the rhesus monkey with emphasis on the external carotid system. Am. j. anat. 16(1):149-170.

CONROY, G.C. \& PACKER, D.T. 1981. The anatomy and plulogenetc significance of the carotid arteries and nevers in Strepsirhine primates. Folia primatol. 35(4):237-247.

DEPEDRINI, J.S. \& CAMPOS, R. 2003. A systematic study of the brain base arteries in the Pampas Fox (Dysicyon Gymnocercus). Braz. j. morphol. sci. 20(3):181-188.
DE VRIESE, B. 1905. Sur la significtion morfologique des artères cerebrales. Arch. biol. 21:357-457.

DING, Y., SHAO, B. \& WANG, J. 2007. The arterial suplly to the brain of the Yak (Bos Grunniens). Ann. anat 189(1):31-38.

DYRUD, J. 1944. The external carotid artery of the rhesus monkey (Macaca mulatta). Anat. rec. 9:17-22.

ELZE, C. 1910. Urber das verhalten der arteria basilaris bei verschiedenen species des Genus ateles. Anatomischer Anzeiger. 37(1):33-38.

FAWCETT, E. \& BLANCHFORD, J.V. 1905-1906. The circle of Willis: an examination of 700 specimens. J. anat. physiol. 40:63-70.

FEAGLE, J.G. 1988. The brain and senses. In: Primate adaptation \& evolution (Feagle J.G.). Academic Press, San Diego, p. 21-23.

FERREIRA, C.G. 2002. Origem trajeto, ramificação e distribuição da artéria cerebral rostral em suínos (Sus scrofa domésticus, Linnaeus, 1758). Tese de doutorado, Universidade de São Paulo, São Paulo.

FERREIRA, J.R, ABREU, N.H.L., PIRES, J.S. \& RIBEIRO, B.N. 2005. O sistema carótico do encéfalo de primata neotropical, anatomia da artéria Inter-hemisférica (Cebus apella, Linnaeus, 1766). Cienc. anim. bras. 6(3):203-212.

FERREIRA, J.R. \& PIRES, J.S. 2004. Estudo anatômico dos ramos da artéria cerebral rostral destinados a região olfatória do encéfalo de primatas neotropicais (Cebus apella, Linnaeus, 1766). Vet. not. 10(2):17-23.

FERREIRA, J.R. \& PRADA, I.L.S. 2001. Nomenclatura proposta para denominar as arterias da base do encéfalo do macaco-prego (Cebus apella L., 1766). Acta sci. 23(2):635-643.

FERREIRA, J.R. \& PRADA, I.L.S. 2003. O circuito anastomótico arterial da base do encéfalo do Macaco Prego (Cebus Apella L., 1766). Vet. not. 9(1):13-36

GILLILAN, L.A. 1969. The arterial and venous blood supplies to the cerebelum of primates. J. neuropathol. exp. neurol. 28(4):295-307.

GUERIN, J., GOUAZE, A. \& LAZORTHES, G. 1976. Le polygone de Willis de l'enfant et les facteurs de son modelage. Neurochir. 22(32):17-26.

HILL, W.C.O. 1960. Primates Comparative Anatomy and Taxonomy; IV Cebidae; Part A. University Press, Edimburg, p. 63-66 e 281-282.

HIMWICH, W.A. \& CLARK, M.E. 1968. Model studies of the circle of Willis: flow and pressure changes. Prog. brain res. 30(2):135-143.

KASSEL, N.F. \& LANGFITT, T.W. 1965. Variations in the circle of Willis in Macaca mulatta. Anat. rec. 62(4):257-264.

KRAMER, S.P. 1912. On the function of the circle of Willis. J. exp. med. 15:348-355.

KRISHNAMURTI, A. 1968. The cerebral arteries of Nycticebus Couang couang. Folia primatol. 8(2):159-168.

LINDERMAM, T., RECKZIEGEL, S. \& CAMPOS, R. 2000. A systematic study of brain Base arteries in the Opossum Didelphis Albiventris. Braz. j. morphol. sci.17(1):35-41.

LINEBACK, P. 1961. The vascular system. In: The anatomy of the rhesus monkey. (C. H. Hafner, eds). C.H. Hartman, New York, p. 255-281.

McKENNA, M.C. 1966. Paleontology and the origin of the primates. Folia primatol. 4(1):1-25.

MELO, A.P.F. 1996. Estudo anatômico das artérias da base do encéfalo em fetos de bovinos azebuados. Dissertação de Mestrado, Faculdade de Medicina Veterinário e Zootecnia da Universidade de São Paulo, São Paulo.

MIRAGLIA, T. \& TEIXEIRA, A.M. 1960. Apuunti sulla struttura e sulla vascolarizzazione dell'ipofise dell sagüi (Callithrix Jacchus). Excerpta med. 13(3):199-218.

PIRES, J.S. \& FERREIRA, J.R. 2004. Anatomia das artéria pericalosas do encéfalo de primata neotropical e seus ramos frontais e parietais (Cebus apella, Linnaeus, 1766). Braz. j. vet. res. anim. sci. 41(3):207-214.

PUCHARDES-ORTS, A., NOMBLELA-GOMES, M. \& ORTUNOPACHECO, G. 1975. Variation in form of circle of Willis: some anatomical and embryological considerations. Anat. rec. 185(2):119-124. 
RAVEN, H.C. \& HILL, J.H. 1950. Regional anatomy of the Gorilla. In: The anatomy of the Gorilla. (H.C. Raven), Columbia University Press, New York, p. 24-26 e 114,115.

RECKZIEGEL, S.H., SCHENEIDER, F.L., EDELWEISS, M.I.A., LINDEMANN, T. \& CULAU, P.O.V. 2004. Anatomy of the caudal cerebral artery on the surface of capybara (Hydrochoerus hydrochaeris) Brain. Braz. j. morphol. sci. 21(3):131-134.

ROGERS, L. 1947. The function of the circulus arteriosus of Willis. Brain. 70(2):171-178.

SANTOS, A. 1987. Estudo anatômico das artérias da base do encéfalo de eqüinos da raça puro sangue inglês. Dissertação de mestrado, Faculdade de Medicina Veterinária e Zootecnia da Universidade de São Paulo, São Paulo.

SHELLSHEAR, T.L. 1927. The arteries of the brain of the Orang-Utan. J. anat. 61(2):167-197.

SHELLSHEAR, T.L. 1930. The arterial supply of the cerebral córtex in the Chympanzee (Anthropopithecus troglodytes). J. anat. 65(1):44-93.

SILVA, R.A. \& FERREIRA, J.R. 2005. O padrão arterial do cerebelo do macaco prego (Cebus apella, L. 1766). Vet. not. 11(2):11-18.

SILVA, R.A. \& FERREIRA, J.R. 2002. Estudo das artérias cerebelares do macaco prego - considerações sobre a nomenclatura (Cebus apella, $L$. 1766). Braz. J. vet. res. anim. sci. 39(6):296-300.

SILVA, R.A. \& FERREIRA, J.R. 2002. Morfologia da artéria cerebelar superior do macaco prego (Cebus apella. L. 1766): divisões e anastomose. Acta sci. 24(3):687-695.

SILVA, R.A. \& FERREIRA, J.R. 2003. Estudos anatomicos das arterias cerebelares inferiores rostrais do macaco prego (Cebus apella, Linnaeus, 1766. Primata - Cebidae). Publicações Avulsas do Instituto Pau Brasil de História Natural. 7(1):15-23.
SILVA, R.A., FERREIRA, J.R. \& PRADA, I.L.S. 2003. Estudo anatômico das artérias cerebelares inferiores caudais do macaco prego (Cebus Apella L. 1766). Arq. cienc. saude UNIPAR. 7(2):107-112.

SIQUEIRA NETO, E.G.B. \& FERREIRA, J.R. 2002. Estudo anatômico da origem e distribuição dos ramos corticais das artérias cerebrais caudais do encéfalo do macaco prego (Cebus apella L., 1766). Acta sci. 24(2):639-646.

SOUZA, M.M.G., TEIXEIRA, A.M.C. \& MIRAGLIA, T. 1962. Dados sobre a vascularização do encéfalo do sagüi (Callinthrix jacchus). Folia clin. biol. 31(2):104-114.

SPERINO, G. 1897. Anatomia del chimpanzé. Unione Tipográfico-Editrice, Torino. p. 264-267.

TESTUT, L. \& LATARJET, A. 1954. Tratado de anatomia humana. Salvat Editora, Rio de Janeiro, p. 1239-1244.

VIEIRA, R.M. 1981. Artéria cerebral anterior do homem: estudo da anatomia de seus segmentos e ramificações. Tese de doutorado, Escola Paulista de Medicina, São Paulo.

WARWICK, R. \& WILLIAMS, P.L. 1979. Gray Anatomia. Guanabara Koogan, Rio de Janeiro, p. 600-605.

WATTS, J.W. 1934a. Ligations of the anterior cerebral artery in the monkey. j. nerv. ment. dis. 79(2):153-158.

WATTS, J.W. 1934b. A comparative study of the anterior cerebral artery and the circle of Willis in primates. J. anat. 68(6):534-550.

WEINSTEIN, J.D. \& HEDGES Jr., T.R. 1962. Sendies of intracranial and orbital vasculatrures of the rhesus monkey (Macaca mulatta). Anat. rec. 144(1):37-41.

Recebido em 11/12/07

Versão reformulada recebida em 15/12/08

Publicado em 31/03/09 